\title{
Injection of a self-consistent beam with linear space charge force into a ring
}

\author{
J. A. Holmes, T. Gorlov, N. J. Evans, M. Plum, and S. Cousineau \\ Oak Ridge National Laboratory, One Bethel Valley Road, Oak Ridge, Tennessee 37831, USA
}

(Received 15 May 2018; published 17 December 2018)

\begin{abstract}
One major concern in the design and operation of high-intensity rings is beam loss due to beam halo. Another issue of importance in applications involving fixed targets is the uniformity of the beam energy deposition on the target. Both of these issues could be favorably addressed by a hard-edged beam with uniform transverse density. This paper presents a detailed feasibility study for painting such a beam into a high-intensity proton ring. For the purposes of this paper, we define self-consistent beams to be ellipsoidal, or elliptical in 2D, distributions that have uniform density and linear space charge force and that retain these properties under all linear transformations. Because of their linear space charge forces and linear transport properties, self-consistent distributions may undergo very little halo formation if realized in practice. Because of their uniform density, they would have smaller maximum space charge tune shifts than peaked density distributions, and they would be attractive for high-intensity fixed target applications. Selfconsistent distributions involve very special relationships between the phase space coordinates, making them singular in some respects and difficult to realize experimentally. The most famous self-consistent distribution is the Kapchinsky-Vladimirsky distribution, but now many other self-consistent distributions have been discovered. One such, the 2D rotating distribution, can be painted as a coasting beam into a ring having an appropriately designed and tuned lattice. For bunched beams, if the bunch length is sufficiently long, it is expected that the coasting beam assumption will be a good approximation during painting. However, it is unknown how robust self-consistent distributions will be under real world transport in the presence of nonlinearities and collective effects. This paper studies these issues for a particular case of interest by applying realistic detailed computational models to the simulation of painting a self-consistent rotating beam into the Spallation Neutron Source (SNS) ring. As a result, we propose a case that can be carried out with only a minor modification of the SNS hardware.
\end{abstract}

DOI: 10.1103/PhysRevAccelBeams.21.124403

\section{INTRODUCTION}

Our motivation in pursuing the work described in this paper is to assess the possibility of injecting a uniform elliptical beam distribution with linear space charge forces into a high-intensity ring. By beam distribution, we mean the coordinates of a collection of particles in $2 \mathrm{~N}$-dimensional phase space which evolve according to the Boltzmann equation, which in turn reduces to the Vlasov equation in the Hamiltonian limit when collisions are neglected [1]. Although all these distributions are defined in $2 \mathrm{~N}$-dimensional phase space, we will refer to them by their coordinate space dimensionality $N$ here. Ideally, the distribution that we want to inject is a $2 \mathrm{D}$, meaning $N=2$, function of two constants of the motion which correspond to the energy and angular momentum for time-independent

Published by the American Physical Society under the terms of the Creative Commons Attribution 4.0 International license. Further distribution of this work must maintain attribution to the author(s) and the published article's title, journal citation, and DOI.
Hamiltonians. It is characterized by an elliptical shape with uniform density and a sharp boundary in the transverse plane and, consequently, by linear internal space charge forces. Furthermore, this distribution retains these properties under all linear transformations. Our interest in injecting such a distribution is motivated by the possibility that its sharp boundaries will lead to a small beam halo and consequent beam loss and that its uniform density will result in low peak beam deposition in fixed target applications.

The assumptions of an ellipsoidal shape and uniform charge density have been used in many analytical and computational studies in beam dynamics. Beam distributions satisfying and maintaining these constraints during transport are an idealized construct. For the present purposes, we will call such distributions self-consistent. The term "selfconsistent" is usually taken to include other distributions, such as the Maxwell-Boltzmann, that are functions of invariants of motion such as the Hamiltonian. However, because our interest is in hard-edged, uniform density distributions with linear space charge forces, we will use the more restrictive definition given here. Self-consistent distributions are functions of invariants of the motion and, for time-independent 
Hamiltonians, are equilibrium solutions of the Vlasov equation. We call invariants time dependent when they depend not only on coordinates and momenta but also directly on time. One example is the Courant-Snyder invariant, which is a quadratic form of the coordinates and momenta with timedependent coefficients. In addition to linear space charge forces that lead to analytic tractability, self-consistent distributions manifest a number of desirable properties. In channels where they are matched, their transport may lead to little halo formation. Because they have uniform density, they would have smaller maximum space charge tune shifts than peaked density distributions, and they would be attractive for highintensity fixed target applications. Furthermore, some selfconsistent distributions can be manipulated to generate flat beams. However, self-consistent beams are not easy to realize in nature.

Kapchinsky and Vladimirsky obtained the first selfconsistent time-dependent space charge distribution (KV distribution) in the two-dimensional case [2]. The KV distribution depends on only one invariant function of coordinates and momenta, which corresponds to a single Hamiltonian when the focusing is time independent, and it takes the form of a uniformly populated constant energy shell in phase space. It was shown in Appendix A in Ref. [3] that an extension of this model to three dimensions is not possible. There is no distribution function in three dimensions that depends on only one invariant (the Hamiltonian in the time-independent case) and produces linear space charge forces. Furthermore, it was shown in Ref. [4] that the KV distribution can be unstable in linear transport channels, and this could be a problem for other self-consistent distributions, too.

Later, methods to obtain self-consistent time-independent space charge distributions were developed for both the cases of linear [5] and nonlinear [6] external focusing and space charge forces. Interesting equations for nonlinear transport of one-dimensional distributions with constant charge density in phase space were analyzed in Ref. [7]. The generation of self-consistent distributions for the general time-dependent case is beyond the reach of modern theoretical mechanics. Even single-particle motion under nonlinear time-dependent forces is not analytically solvable. Exceptions occur in some special cases of forces with symmetries, as in the case of round beams with a special dependence of the force on the time and coordinates (see [8] and references therein).

Round or spherical beams yield the first examples of more general self-consistent time-dependent space charge distributions with linear forces, where the dependence is now on two invariants, one of which corresponds to the angular momentum. Reference [9] shows how to construct such distributions, including cases without special symmetries. This paper was primarily a mathematical exercise. It demonstrated the existence, techniques for constructing, and classification of numerous self-consistent distributions in $\mathrm{N}$ dimensions. It described the mathematical form of the distributions, which could be written as functions of constants of the motion multiplied by products of delta functions involving linear relationships between the generalized coordinates. It proved the preservation of selfconsistency in all linear transformations, and it constructed envelope equations for self-consistent distributions in $2 \mathrm{D}$ and 3D. This paper did not analyze the stability of the newly described self-consistent distributions, but it did pave the way for such studies and for the consideration of practical applications of self-consistent beams in existing or planned accelerators.

\section{HOW TO PAINT A LONG-BUNCH SELF-CONSISTENT BEAM INTO A RING}

An essential consideration for the practical application of self-consistent beams is how to create them. Reference [10] proposed a method to produce a $3 \mathrm{D}$ ellipsoidal uniform density self-consistent distribution of electrons by uniform expansion of a pancake distribution obtained by shining a properly shaped femtosecond laser pulse on a photoemitting cathode. This scheme was later carried out experimentally with some success [11]. However, the generation of a self-consistent distribution in a high-intensity proton ring is a very different problem. In such machines, bunches are typically long and for many purposes are more readily visualized as coasting beams than as spherical distributions. The beam dynamics can be separated into longitudinal (synchrotron) and transverse (betatron) motion that occur on very different timescales. Typically, betatron timescales are orders of magnitude shorter than those for synchrotron motion. In the Spallation Neutron Source (SNS), for example, betatron tunes are approximately 6.2, while the synchrotron tune is about $7 \times 10^{-4}$, a difference of 4 orders of magnitude. Beam distributions are built up in high-intensity proton rings over many turns by painting an incoming $\mathrm{H}^{-}$beam into the circulating proton beam through stripper foil injection. Desired beam distributions in the ring can be obtained by programming the time dependence of the injection parameters.

With all this in mind, an excellent candidate distribution for painting into a ring was identified in Ref. [9]. Longitudinally, the distribution is uniform, so that the distribution's selfconsistency comes from its transverse properties. In the nomenclature of Ref. [9], it was classified as a $\{2,2\}$ distribution, meaning a 2D distribution (4D in transverse phase space) with two linear relations between phase space variables expressed as delta functions, one relating $x$ to $y^{\prime}$ and the other relating $y$ to $x^{\prime}$. In this distribution, there are two constants of motion, which reduce to the energy and angular momentum in the time-independent case. The notation $\{n, m\}$ means $\mathrm{n}$ dimensions and $\mathrm{m}$ delta functions. The $\{2,2\}$ distribution is commonly called a rotating distribution, because the beam rotates in the transverse plane. The rotating distribution can be painted as a coasting beam into a ring, but 
three conditions must be satisfied: (i) The painted horizontal and vertical emittances must increase linearly in time from initial values of zero to yield a constant charge density; (ii) the painted horizontal and vertical phases need to differ by $90^{\circ}$ to obtain a rotating elliptical distribution in transverse space; and (iii) the vertical and horizontal tunes have to be equal so that the beam can rotate by maintaining a constant relationship between the horizontal and vertical particle phases, at least on a turn-by-turn basis. The first condition determines the time dependence of a painting scheme in which

$$
\begin{aligned}
x_{\mathrm{inj}}-x_{\mathrm{CO}} & =x_{\mathrm{Max}} \times \sqrt{t / t_{\mathrm{Max}}} \mathrm{mm}, \\
x_{\mathrm{inj}}^{\prime}-x_{\mathrm{CO}}^{\prime} & =x_{\mathrm{Max}}^{\prime} \times \sqrt{t / t_{\mathrm{Max}}} \mathrm{mrad}, \\
y_{\mathrm{inj}}-y_{\mathrm{CO}} & =y_{\mathrm{Max}} \times \sqrt{t / t_{\mathrm{Max}}} \mathrm{mm}, \\
y_{\text {inj }}^{\prime}-y_{\mathrm{CO}}^{\prime} & =y_{\mathrm{Max}}^{\prime} \times \sqrt{t / t_{\mathrm{Max}}} \mathrm{mrad},
\end{aligned}
$$

where the subscripts inj and $\mathrm{CO}$ refer to injection and closed orbit, respectively. The second condition determines the relative sizes of $x_{\text {Max }}$ and $y_{\text {Max }}^{\prime}$ and separately of $y_{\text {Max }}$ and $x_{\text {Max }}^{\prime}$. There is still a freedom of choice of phase that allows different proportions of $x-y^{\prime}$ and $y-x^{\prime}$ to be painted. It is important to note that, under the above self-consistent painting scheme, the accumulating distribution is selfconsistent throughout the process, not just at the final time.

This painting scheme for the rotating distribution is quite different from that used in most high-intensity rings. For example, the scheme used in SNS production, which is commonly called correlated painting, sets $x_{\operatorname{Max}}^{\prime}$ and $y_{\text {Max }}^{\prime}$ to zero and includes an initial offset so that

$$
\begin{aligned}
& x_{\mathrm{inj}}-x_{\mathrm{CO}}=\left[\left(x_{\mathrm{Max}}-x_{0}\right) \times \sqrt{t / t_{\mathrm{Max}}}+x_{0}\right] \mathrm{mm}, \\
& y_{\mathrm{inj}}-y_{\mathrm{CO}}=\left[\left(y_{\mathrm{Max}}-y_{0}\right) \times \sqrt{t / t_{\mathrm{Max}}}+y_{0}\right] \mathrm{mm} .
\end{aligned}
$$

Correlated painting tends to create rectangular distributions in real space.

\section{REALISTIC SIMULATIONS OF ROTATING SELF-CONSISTENT BEAMS}

A successful simulation of the injection of a 2D selfconsistent rotating distribution as a coasting beam, including space charge, into a linearized SNS lattice was conducted using the ORBIT code [12] and presented in Ref. [9]. The simulation demonstrated that all three of the conditions for self-consistent painting had to be satisfied to obtain a selfconsistent distribution. When we say that a beam in a simulation is self-consistent, we mean that it has an elliptical or ellipsoidal shape and uniform density and that any linear relationships between phase space variables are propagated through the lattice. A central question in the study of selfconsistent beams is their robustness under real transport conditions including space charge, nonlinearities, impedances, bunched beam properties, and other loss mechanisms.
A computational approach is an effective way to address this question, with an emphasis on systematically introducing increasingly realistic effects: chromaticity and nonlinearities, fringe fields, foil scattering and collimation, impedances, bunched beams, and real injection (as opposed to artificial bumps). When realistic physics is included, we expect self-consistent properties to be blurred to some extent, and it is necessary to determine the degree of selfconsistency in the resulting distribution. To do this, the distribution shape and density in phase space can be examined; beam profiles can be compared with mathematically self-consistent profiles; average incoherent tunes in the transverse plane can be calculated and compared; and correlations between different phase space variables can be calculated. The correlation coefficient between two variables $\mathrm{u}$ and $\mathrm{v}$ is defined by

$$
c_{u v}=\frac{\langle(u-\langle u\rangle) *(v-\langle v\rangle)\rangle}{\sqrt{\left\langle(u-\langle u\rangle)^{2}\right\rangle\left\langle(v-\langle v\rangle)^{2}\right\rangle}},
$$

where the brackets denote averages over the distribution. The values of coefficients of uncorrelated variables are near zero, and strongly correlated variables have coefficients approaching \pm 1 depending on a positive or negative slope. All of these properties are easy to test in simulations, and we do so routinely in our studies. We now discuss selfconsistency when realistic physical effects are included in the calculations.

As stated above, it is possible to paint a coasting selfconsistent rotating beam into a linearized lattice, either with or without space charge. The introduction of nonlinearities, including chromaticity, multipoles, and fringe fields, was found to destroy rotating beam self-consistency by coupling degenerate eigensolutions, whose superposition is not self-consistent (Fig. 1, left). In a linear $x-y$ decoupled lattice having equal tunes, there are four independent degenerate rotating beam eigenfunctions. Two solutions correspond to beams rotating clockwise, and the other two correspond to beams rotating counterclockwise. The two independent eigenfunctions corresponding to either direction of rotation differ only in phase. While these corotating eigenfunctions can be superposed to yield a rotating selfconsistent beam, the superposition of oppositely rotating eigenfunctions destroys self-consistency, as the resulting distribution is no longer a function of angular momentum.

The solution is to break the rotating or counterrotating degeneracy. To do this, solenoid magnets can be introduced in order to defocus one of the rotating eigenfunctions and to focus the counterrotating beam, thus splitting their respective tunes. The presence of the solenoids leads to two pairs of complex conjugate eigenfunctions, each with its own pair of complex conjugate eigenvalues, for the ring transfer matrix in transverse phase space. It is possible to paint a self-consistent rotating distribution to the real part, the imaginary part, or any linear combination thereof, for either 

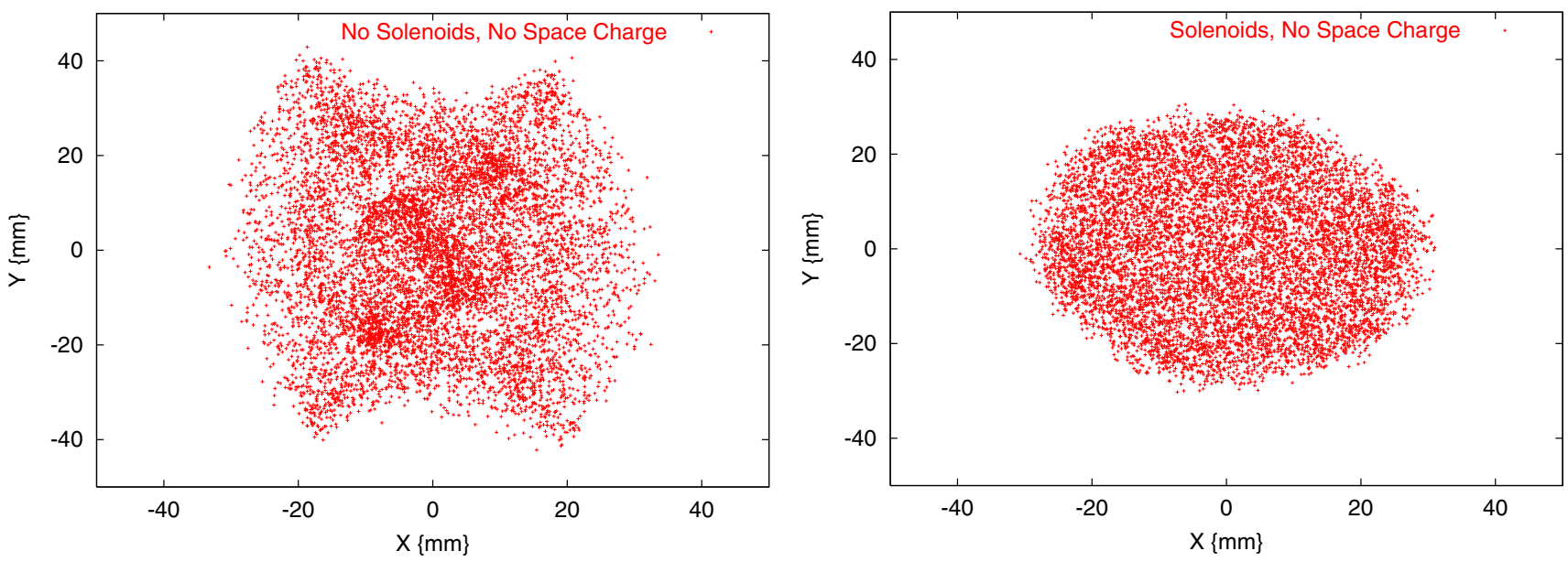

FIG. 1. Results of painting into a rotating beam eigenmode with linearly increasing emittance. (Left) Degenerate eigenfunctions couple. (Right) Solenoids can be added to the lattice to decouple the eigenfunctions.
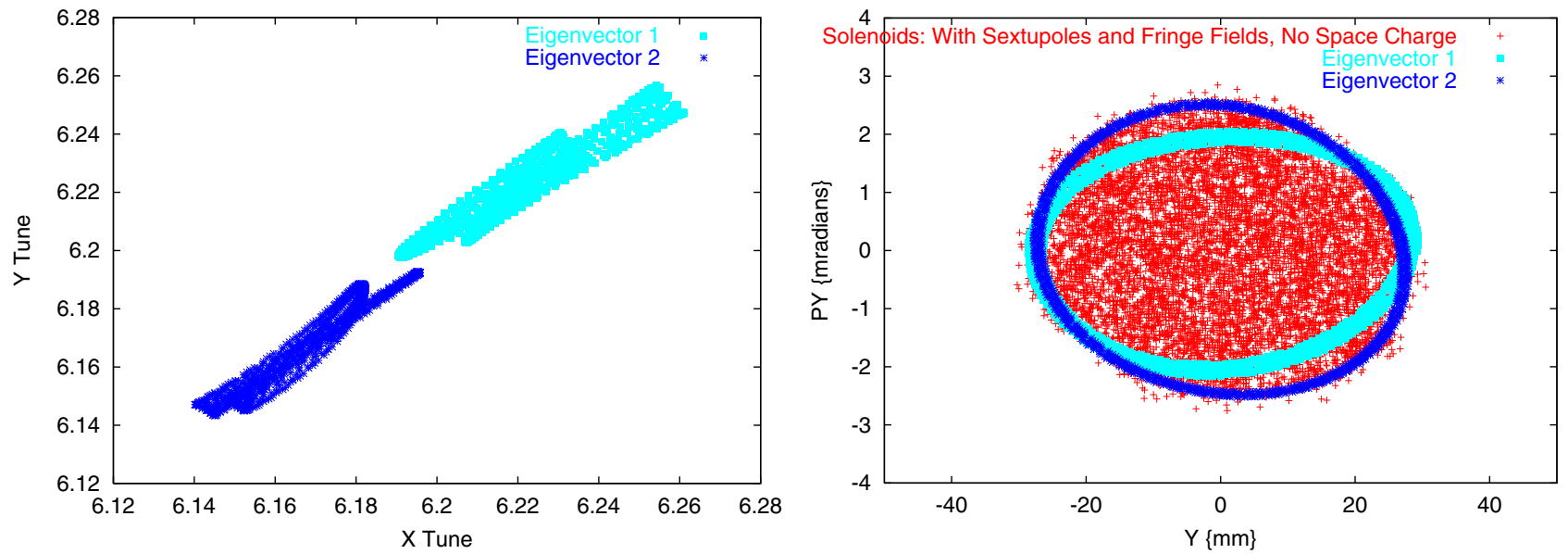

FIG. 2. (Left) Tune footprint for the two eigenvectors, each taken turn by turn over 1000 turns. (Right) Eigenvector projections in vertical phase space, together with self-consistent beam distribution painted to one (dark blue) of the eigenvectors.

of the eigenfunction pairs (Fig. 1, right, and Fig. 2). Figure 2 illustrates the splitting of the tunes of the rotating and counterrotating beams (left) together with the ability to paint into a chosen eigenfunction (right). In summary, solenoid magnets can be used to maintain self-consistency when there are nonlinearities by splitting the tunes of the rotating and counterrotating beams, thus allowing injection into either of the two eigenfunction pairs.

Another important issue is the effect of bunched beams. Most high-intensity rings are not operated with coasting beams. If the bunch is sufficiently long that transverse beam properties change slowly along the longitudinal direction, it might be expected that a coasting beam will be closely approximated. For example, in the SNS, the length of the beam in the ring is more than $150 \mathrm{~m}$, and the coasting beam approximation should be acceptable as long as the longitudinal density is kept uniform. Even though the synchrotron tune is typically orders of magnitude less than the betatron tunes, the longitudinal evolution of a bunch during accumulation, acceleration, and storage is significant. As a computational experiment, we carried out two bunched beam simulations using the SNS lattice. In one, we used the design voltages of the SNS dual harmonic bunchers. In the other, we replaced the dual harmonic rf cavities by barrier cavities, which maintain a nearly constant density longitudinal profile. Simulations with the barrier cavities showed that it is possible to inject a chopped rotating self-consistent beam into the SNS ring. This was not true when the design voltages of the SNS cavities were employed. The design dual harmonic and assumed barrier cavity waveforms are shown in the left-hand plot in Fig. 3, and the resulting beam current profiles following full design-intensity injection simulations are shown in the right-hand plot. The barrier cavity current profile is much flatter than the current profile from the dual harmonic case. The important information that these simulations provided is that it is possible to paint 

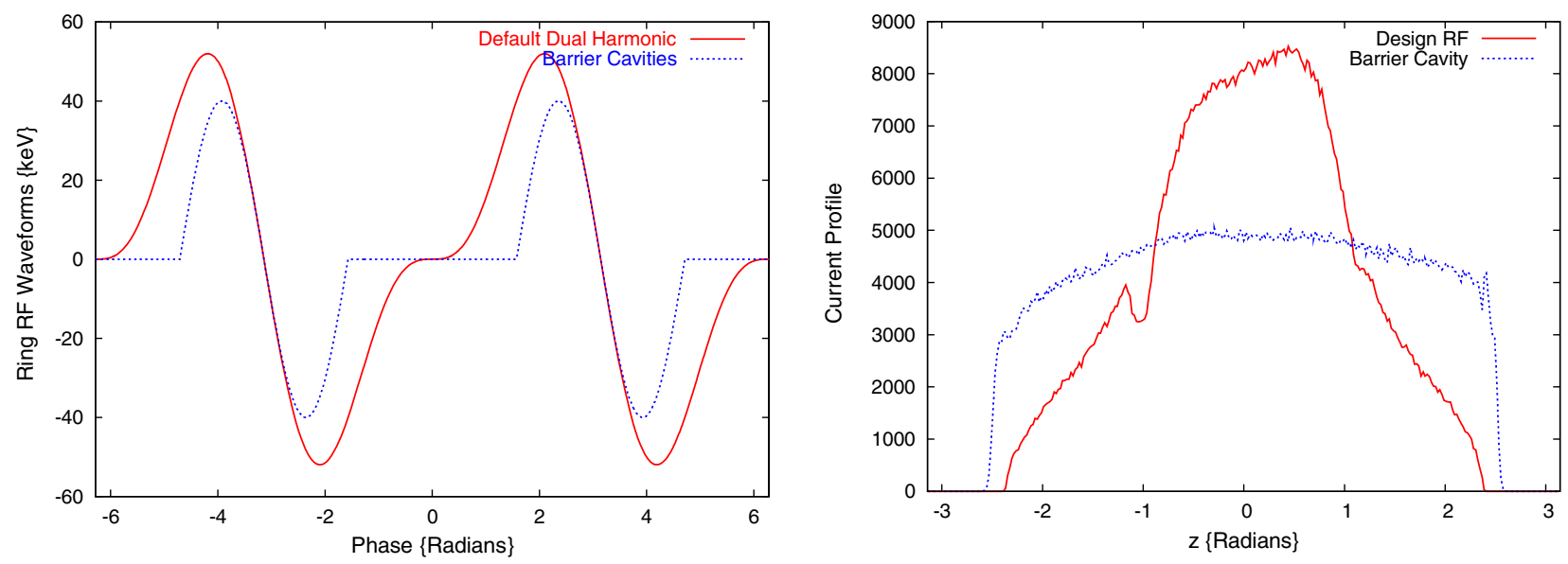

FIG. 3. (Left) Design dual harmonic and assumed barrier cavity waveforms plotted for two periods. (Right) Beam current profiles following accumulation for dual harmonic and barrier cavity rf waveforms. The bunch longitudinal coordinate $z$ is normalized to the ring length divided by $2 \pi$ so that the full scale represents $248 \mathrm{~m}$ in these simulations.

a rotating distribution into a bunched beam if the longitudinal distribution is kept uniform but that failure to do so can make this impossible. The results of the computational work described above have been published in conference proceedings [13-16].

At 40 and $20 \mathrm{kV}$ first and second harmonic voltages, respectively, the design $\mathrm{rf}$ bunching in the SNS ring is very strong. Today, the ring rf maintains the beam gap with much smaller voltages than the design values, well under $10 \mathrm{kV}$ in both the first and second harmonics. Such low voltages should lead to much less distortion of the longitudinal current profile than occurs at the design values. In order to study a bunched beam injection intermediate to those with barrier cavities and with the SNS design values, we have carried out the same injection simulation but now using the dual harmonic rf with settings of $4 \mathrm{kV}$ in both the first and second harmonics. These values are in the range of

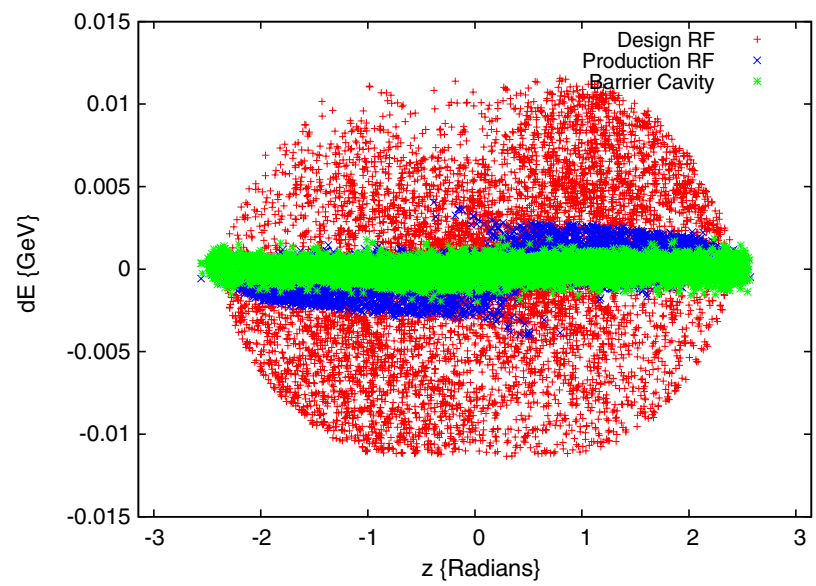

present production voltages and are sufficient to maintain the beam gap. The resulting longitudinal beam profile following full-intensity injection was somewhat more peaked than that obtained with barrier cavities but still quite broad and uniform when compared to that resulting from the design rf voltages. Figure 4 summarizes the longitudinal behavior for three rf scenarios: dual harmonic rf at the design voltages of $40 \mathrm{kV}$ first harmonic and $20 \mathrm{kV}$ second harmonic (in red), the barrier cavity waveform shown in Fig. 3 (in green), and dual harmonic rf with $4 \mathrm{kV}$ in both harmonics (in blue). In the transverse plane, the injected distribution retained its selfconsistency using the $4 \mathrm{kV}$ rf settings. Therefore, we have adopted the $4 \mathrm{kV}$ rf settings for all subsequent calculations using the SNS lattice, including those in this paper.

The results presented above utilize an artificial bump method to carry out the injection painting. In ORBIT's artificial bump method, the injection kickers are treated as

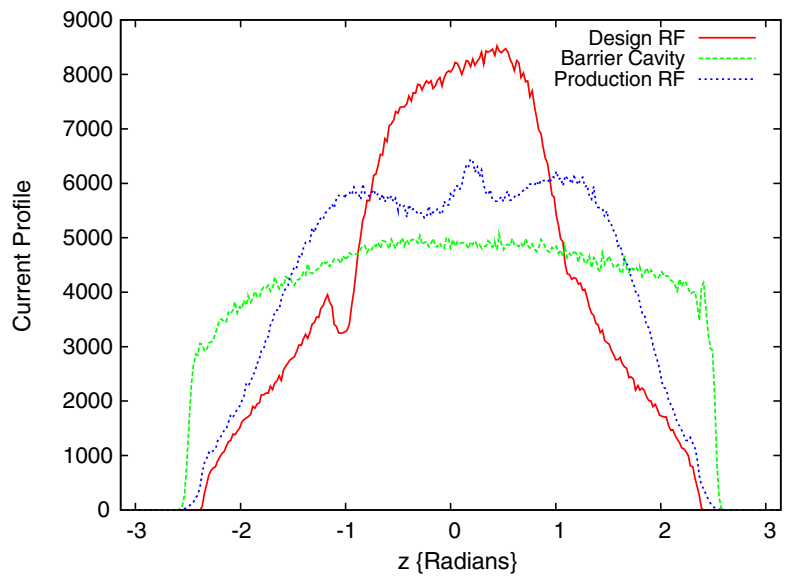

FIG. 4. Results following accumulation at full intensity are shown for three rf bunching scenarios: dual harmonic rf at the SNS design voltages of $40 \mathrm{kV}$ first harmonic and $20 \mathrm{kV}$ second harmonic (red), the barrier cavity waveform shown in Fig. 3 (green), and dual harmonic rf with $4 \mathrm{kV}$ in both harmonics (blue). (Left) Longitudinal phase space distributions and (right) longitudinal current profiles for the three cases. 

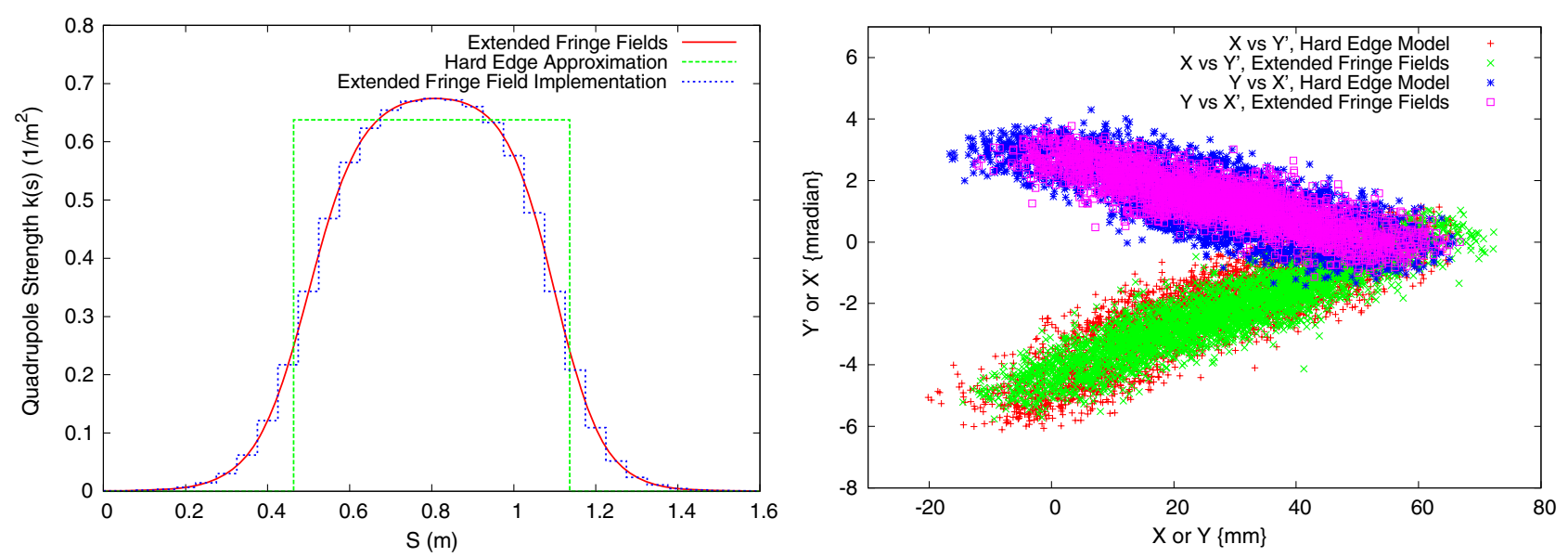

FIG. 5. (Left) Quadrupole strength functions for the hard-edge model (green), for an extended fringe field model (red), and for oRBIT's extended fringe field implementation (blue). The integrated areas under the three curves are equal. (Right) Comparison of beam distributions for a painted self-consistent beam using the hard edge (red and blue) and orBIT's extended (green and pink) fringe field models.

drifts, and the closed orbit passes through the centers of all lattice elements. The injected beam is added at the azimuthal location of the stripper foil with the correct transverse coordinates relative to the closed orbit. With the full injection painting scheme, the beam is kicked at the actual kicker locations and settings including the appropriate time dependence of the painting scheme, and the injected beam is added at the actual foil location in both planes. The main difference between these two schemes is that, in the injection straight section the closed orbit passes through the element centers in the artificial bump model, but it encounters the elements off center in the real kicker model. There are two main consequences of using the real kicker model, and both are a result of the off-axis beam transport. The first consequence regards fringe field effects. We consider these here in the context of the SNS lattice, but similar considerations are certainly present in other machines. In the SNS ring, there are two quadrupole doublets in the injection straight section, and the kicked beam encounters those magnets well off center. When quadrupole fringe fields are included in the calculations, their effects could become significant, because the strength of their contribution increases off axis. In order to assess the limitations associated with the hard-edge fringe field approximation in the injection region and to more correctly evaluate the effects of the fringe fields on the off-axis beam, we developed an extended fringe model based on the assumption that the quadrupole field strength varies along the magnet axis. According to this model, the vector potential of a quadrupole magnet can be represented as

$$
\begin{aligned}
& A_{x}=-k^{\prime} / 12^{*}\left(x^{3}+3 x y^{2}\right)-k_{s}^{\prime} / 6^{*} y^{3}+O(4), \\
& A_{y}=+k^{\prime} / 12^{*}\left(3 x^{2} y+y^{3}\right)-k_{s}^{\prime} / 6^{*} x^{3}+O(4), \\
& A_{s}=k / 2^{*}\left(y^{2}-x^{2}\right)-k_{s} x y+O(5),
\end{aligned}
$$

where the quadrupole and skew quadrupole strengths $k$ and $k_{s}$ are functions of the beam line coordinate $s$, the prime symbols denote differentiation with respect to $s$, and the " $O$ " symbols denote the order of the omitted terms in the transverse phase space coordinates. While the usual quadrupole "body" terms appear in the longitudinal component $A_{s}$, the fringe field terms constitute the contributions to the transverse components $A_{x}$ and $A_{y}$. In the hard-edge model, it is assumed that the quadrupole strengths can be represented by step functions, so that the $k^{\prime}$ terms are delta functions. The symplectic integration of these terms in the hard-edge fringe field model in ORBIT is taken from the treatment in Ref. [17]. In order to carry out the integration for extended fringe fields, we conceptually replace the step function descriptions of $\mathrm{k}$ and $k_{s}$ by smooth curves such that the integrated area under the curves gives the total integrated field strength, as shown in red in the left-hand plot in Fig. 5. The implementation of extended fringe fields in ORBIT replaces this smooth representation of $\mathrm{k}$ by a sliced "staircase" model, shown in blue in Fig. 5, in which the evaluation of the body fields on each slice is alternated with a hard-edge fringe field evaluation at each step between slices. This allows for the use of the same algorithms that are employed in the hard-edge model. In the ORBIT extended fringe field model, used here for the quadrupole doublet magnets in the injection straight section, the function $k$ is taken from the OPERA3D/TOSCA [18] calculations in Ref. [19]. A comparison of results obtained using the extended and hard-edge fringe field models in ORBIT is shown in the right-hand plot in Fig. 5. The plot shows, for one particular self-consistent case, the distributions of $y^{\prime}$ versus $x$ and $x^{\prime}$ versus $y$ at the end of injection, both of which should be linearly related in an ideal self-consistent beam. Real effects, including finite injected beam size, lattice nonlinearities, collective effects, ring rf bunching, 

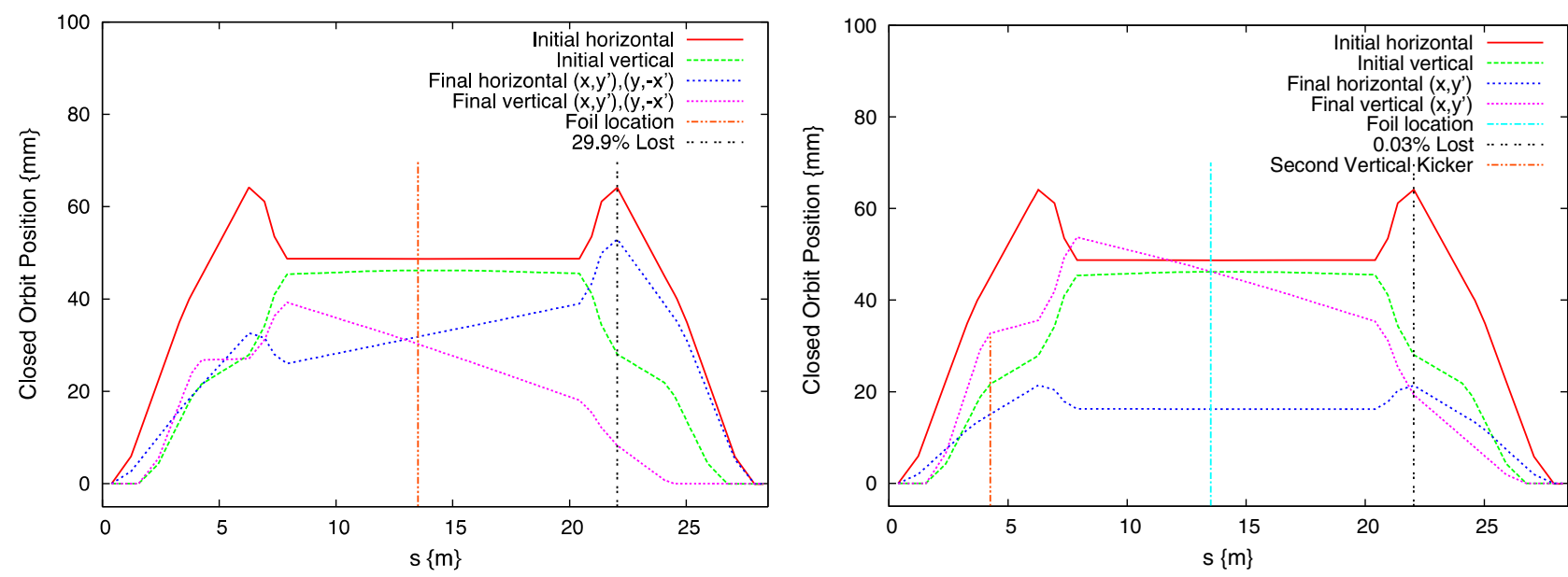

FIG. 6. (Left) Initial and final closed orbit offsets in the injection straight section for a painting scheme with comparable portions of $x-y^{\prime}$ and $y-x^{\prime}$ painting. Note that beam loss is nearly $30 \%$ due to scraping just after the downstream quadrupole doublet. (Right) Initial and final closed orbit offsets in the injection straight section for a painting scheme varying $x-y^{\prime}$ only. Now the beam loss is reduced by 3 orders of magnitude to $0.03 \%$, still due to scraping after the downstream quadrupole doublet.

and foil scattering broaden the distributions into bars. The figure shows that the effect of the extended fringe fields is to reduce the broadening somewhat without causing any major qualitative changes. Henceforth, the results presented here are calculated using extended fringe fields for the doublet magnets in the injection region.

The second consequence of off-axis beam transport is the possibility of beam scraping and resulting beam loss. We again consider this issue in the context of the SNS lattice. In simulations with artificial bumps, the beam traverses the center of the beam pipe and losses are generally acceptable, but with off-axis beam transport beam loss can increase because of scraping. In order to address this issue, the simulations incorporate a detailed description of the machine geometry, including element offsets and apertures, throughout the ring and especially in the injection region. In the SNS, there are two readily available "knobs" that can be applied to minimize beam loss in injection.

One possibility would be to steer the injected beam coming from the linac to a point on the stripper foil closer to the central axis of the injection chicane. By moving this point by $5 \mathrm{~mm}$ in both the horizontal and vertical directions, about the maximum extent possible, simulations predict a significant reduction in the loss rate. However, shifting the injection point by this amount would necessitate a reevaluation and possible redesign of the incompletely stripped waste beam trajectories to the injection dump. These trajectories are very sensitive to the settings of the injection chicane magnets and to the location of the injected beam, and it is not clear that a shift of $5 \mathrm{~mm}$ can be easily accommodated.

Another possible remedy that requires no change to the injected beam position is to take advantage of the freedom of choice in the phase of the rotating beam at the injection point. For example, we could paint with a linear relationship between $x-y^{\prime}$ while holding $y-x^{\prime}$ constant, or vice versa. This corresponds to setting either $y_{\operatorname{Max}}$ and $x_{\operatorname{Max}}^{\prime}$ or $x_{\operatorname{Max}}$ and $y_{\text {Max }}^{\prime}$ to zero in Eq. (1), respectively. We could also paint to the counterrotating eigenfunction, again with freedom of choice of the phase. There are an infinite number of possibilities. We experimented with several of these and found that losses could vary from nearly $100 \%$ when painting purely in $y-x^{\prime}$ to almost zero when painting purely in $x-y^{\prime}$. This is illustrated in Fig. 6, which shows the initial and final bumped closed orbits through the injection region for two different painting phase choices. The $x-y^{\prime}$ painting scheme shown on the right leads to much lower beam loss than the scheme shown on the left, which has comparable portions of $x-y^{\prime}$ and $y-x^{\prime}$ painting. With the exception of the painting waveforms, the lattice settings were the same in all these cases, which led to comparable beam sizes. The variation in beam losses results from steering the beam either closer to or further from the beam pipe and not from a variation in the beam size. The losses in all these painting schemes occur at the focusing quadrupole in either the upstream or downstream doublet, where the horizontal beam offset is large (see Fig. 6, and see also Fig. 10 for the locations of the kickers and the quadrupole doublets). This situation is exacerbated when $x^{\prime}$ is varied in the painting scheme. For this reason, the $x-y^{\prime}$ painting scheme is most favorable with respect to losses so that $y_{\operatorname{Max}}$ and $x_{\text {Max }}^{\prime}$ are set to zero in Eq. (1). Another advantage of the $x-y^{\prime}$ painting scheme, specific to the SNS, is that a reasonable beam size can be obtained without any injection kicker power supplies changing sign. For these reasons, we use the $x-y^{\prime}$ painting scheme for rotating beam injection studies in the SNS.

Although the calculations presented here have been specific to the SNS, similar issues will need to be addressed in considering the injection of a self-consistent beam into 

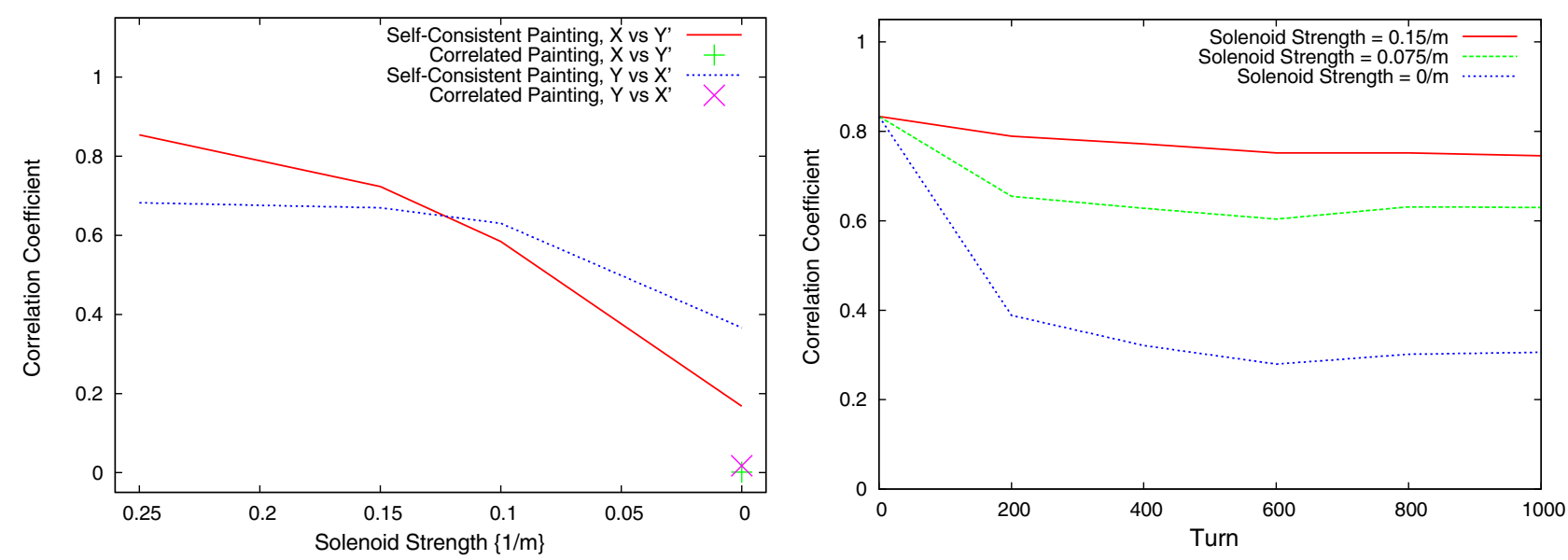

FIG. 7. (Left) Correlation coefficients following injection for $x-y^{\prime}$ and $y-x^{\prime}$ versus solenoid strength for self-consistent painting (red and blue curves) and for correlated painting (green and pink points). On the horizontal scale, a solenoid strength of $0.25 \mathrm{~m}$ corresponds to a magnetic field of $1.414 \mathrm{~T}$ at $1.0 \mathrm{GeV}$. (Right) Correlation coefficients in $x-y^{\prime}$ for different solenoid strengths as functions of the turn number during storage of a previously injected beam for an additional 1000 turns.

any ring. The specific solutions to the issues considered will be machine specific, and it is quite likely that more optimal answers can be found if one were to design a machine for self-consistent injection rather than adapt an existing machine. The important point to be stressed is that simulations using a sophisticated computational representation of an existing machine suggest that it is possible to paint a self-consistent beam into that machine with the addition of solenoid magnets into the lattice. The computational studies now incorporate the full physics modeling capabilities of the ORBIT code together with a detailed description of the SNS accumulator ring lattice. Specifically, the calculations employ symplectic singleparticle tracking with fringe fields for all magnets, including extended fringe field models for two doublet pairs in the injection straight section that are traversed by the beam off axis; detailed models for the injection painting with time-dependent waveforms for all kickers; bunched beams with realistic voltages for the ring rf bunchers; a 2.5D sliced model for longitudinal and transverse space charge; the dominant longitudinal and transverse impedances of the extraction kickers; a realistic parameterization of the injected beam distribution at the stripper foil; scattering of the injected and circulating beams due to the stripper foil; and an extensive set of apertures to model beam losses around the ring.

\section{SENSITIVITY STUDIES: ROBUSTNESS OF SELF-CONSISTENCY}

Having shown that it is possible to paint a self-consistent distribution into a high-intensity ring with realistic simulations, it is of interest to determine how robust selfconsistency is with respect to a number of parameters. Among these are the strength and placement of the solenoid magnets. The initial studies, using a linearized SNS lattice, incorporated two solenoids of length $0.5 \mathrm{~m}$ and strength $1.4 \mathrm{~T}$ that were placed symmetrically at locations of equal vertical and horizontal beta functions in the rf straight section of the ring. In order to assess the possibility of reducing the magnetic field in the solenoids, we carried out identical calculations with two $0.5 \mathrm{~m}$ solenoids, varying only the solenoid field strength. The results are shown in Fig. 7. The left-hand plot shows the magnitudes of the $x-y^{\prime}$ (in red) and $y-x^{\prime}$ (in blue) correlation coefficients at the completion of injection as a function of the solenoid field strength. On the horizontal axis, the value $0.25 \mathrm{~m}^{-1}$ corresponds to a magnetic field of $1.41 \mathrm{~T}$ at a beam energy of $1.0 \mathrm{GeV}$. We see that the correlations indicating selfconsistency remain quite strong down to field strengths of $0.1 \mathrm{~m}^{-1}$. Just for the sake of comparison, the left-hand plot in Fig. 7 also shows the $x-y^{\prime}$ and $y-x^{\prime}$ correlation coefficients following injection for the production SNS correlated painting scheme. As expected, the values are very close to zero. The right-hand plot in Fig. 7 shows the $x-y^{\prime}$ correlation coefficients as functions of the turn number during storage of a previously injected beam for an additional 1000 turns. The calculations are identical except for the solenoid strength. The same initial distribution was used in all three cases. The figure shows that the correlations adjust to levels at which they remain fairly constant and that the correlations decrease with a decreasing solenoid strength. Even though the correlations remain substantial at $0.075 \mathrm{~m}^{-1}$, we have chosen to use a somewhat more conservative value of $0.15 \mathrm{~m}^{-1}$ for the solenoid field strength in the studies presented here. For results at a lower energy, a fixed solenoid field strength means that the corresponding field scales proportionally to the beam momentum.

In order to determine the sensitivity to the number and position of the solenoid magnets, a variety of calculations 


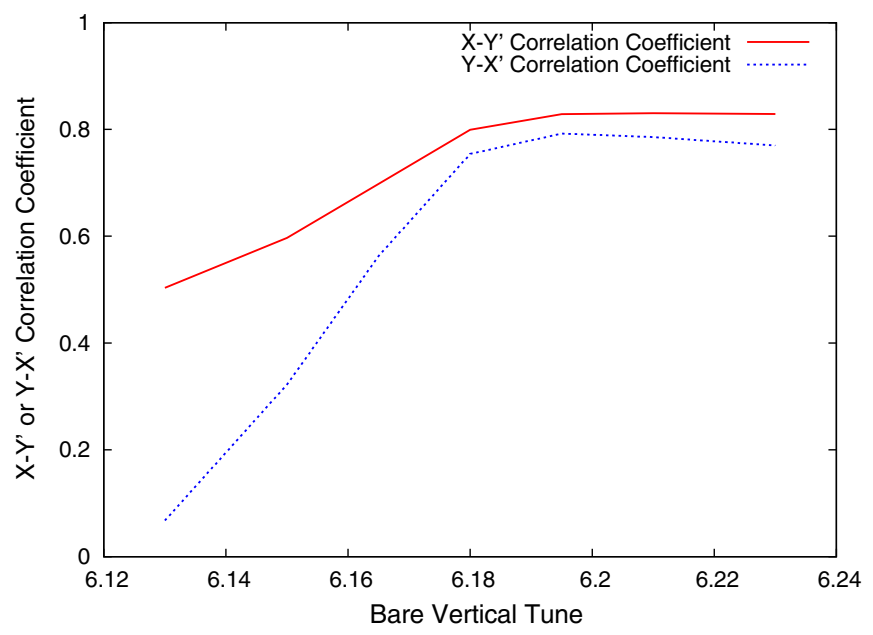

FIG. 8. Correlation coefficients following injection for $x-y^{\prime}$ (red) and $y-x^{\prime}$ (blue) versus $Q_{y}$.

were carried out. In these calculations, the magnets were moved upstream and downstream in various combinations, moved to totally different locations, and finally replaced by solenoids in different numbers, lengths, and locations while maintaining a fixed integrated field strength. The results in all these cases were virtually identical, showing that selfconsistency is sensitive mainly to the total integrated solenoid field strength rather than to the exact configuration of the solenoids.

Another question of interest is the sensitivity of selfconsistency to the $x-y$ tune separation. As stated above, a requirement for painting a rotating self-consistent beam is equality of the horizontal and vertical tunes. In order to determine how precisely this condition must be satisfied by the bare tunes, we carried out a series of injection calculations for 600 turns with horizontal bare tune $Q_{x}=6.18$, identical except for the vertical tune setting. In this study, the vertical bare tune ranged from

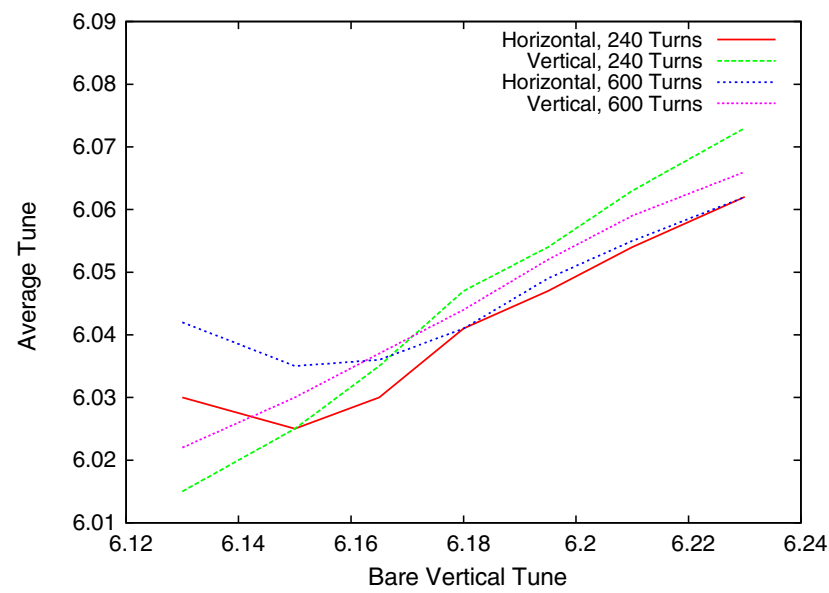

$6.13 \leq Q_{y} \leq 6.23$. The absolute values of the resulting $x-y^{\prime}$ and $y-x^{\prime}$ correlation coefficients following injection are plotted versus the bare tune $Q_{y}$ in Fig. 8. These results show a very strong correlation for tunes above about 6.16, with a decreasing correlation at lower tunes. Some insight into this behavior can be gained by examining the average incoherent tunes of the particles in these cases. The lefthand side in Fig. 9 shows the average horizontal and vertical tunes, plotted versus $Q_{y}$ both at 240 turns and at 600 turns, for these cases. For a perfectly self-consistent rotating beam, these tunes should be equal to each other at all times, and the present results are nearly so for $Q_{y}$ above about 6.16. Note that the average tunes do not equal the bare tunes due to nonlinear and collective effects. On the right-hand side, the difference between average tunes, $\left\langle Q_{y}\right\rangle-\left\langle Q_{x}\right\rangle$, is plotted at 240 turns and at 600 turns. Figure 9 shows that the average horizontal and vertical tunes display very similar behavior when the bare tune $Q_{y}$ is greater than about 6.16, but at lower vertical bare tunes they deviate, as can be seen in the right-hand plot of $\left\langle Q_{y}\right\rangle-\left\langle Q_{x}\right\rangle$ in Fig. 9. Note that the range in which the average tunes differ corresponds to the range in which the self-consistent correlations decrease, indicating the absence of a rotating beam.

The likely explanation for the observed behavior is that, for bare tunes $Q_{y}$ greater than about 6.16, the beam configuration adjusts to maintain equal incoherent tunes and strong self-consistent correlation, even when $Q_{x} \neq Q_{y}$. At smaller values of $Q_{y}$, the beam is moving into the stop band of the half-integer resonance at $Q_{y}=6$, and this becomes the dominant factor in determining the beam configuration. Beam broadening caused by the half-integer resonance at $Q_{y}=6$ is well known and has been studied in Refs. [3,20-22]. In any case, the $Q_{y}$ scan demonstrates that there is a comfortable range of tunes around $Q_{x}=Q_{y}$ in which self-consistent beams can be painted.

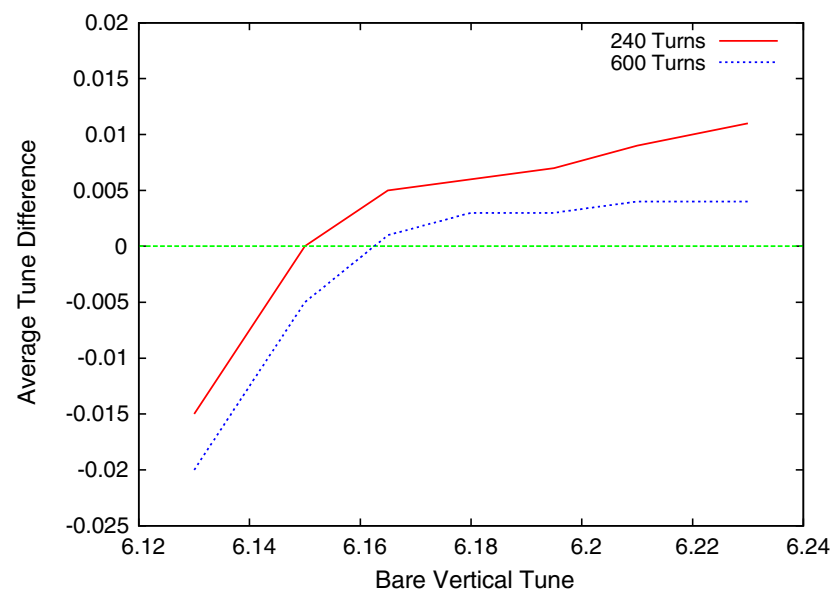

FIG. 9. (Left) Average incoherent horizontal $\left\langle Q_{x}\right\rangle$ and vertical $\left\langle Q_{y}\right\rangle$ tunes versus bare tune $Q_{y}$ at 240 turns (red and green curves) and at 600 turns (blue and pink curves). (Right) Tune difference $\left\langle Q_{y}\right\rangle-\left\langle Q_{x}\right\rangle$ at 240 turns (red curve) and at 600 turns (blue curve). The green line marks $\left\langle Q_{y}\right\rangle-\left\langle Q_{x}\right\rangle=0$, or equal tunes. 


\section{DETAILED CASE FOR PROPOSED EXPERIMENT}

The above results suggest that a rotating self-consistent beam can be painted into a ring, using the SNS as an example, if the longitudinal variation along the bunches is slow in space and in time and if the conditions for injection painting are satisfied. Furthermore, the results suggest that beam self-consistency is achievable over a range of conditions such as solenoid strength and configuration and bare tune settings. Because we are about to propose a case to be realized experimentally in the SNS, we now provide a description of the machine. The SNS ring has a length of $248 \mathrm{~m}$. It consists of four achromatic arcs separated by four straight sections which are used for injection, collimation, extraction, and rf focusing, respectively. In production, a $1 \mathrm{GeV} \mathrm{H}^{-}$beam is injected through a stripper foil into the ring and accumulated for more than 1000 turns, or about $1 \mathrm{~ms}$, until the beam intensity exceeds $10^{14}$ protons. When the desired beam intensity is achieved, the beam is extracted in a single turn and delivered to the target. Longitudinally, a uniform beam having a length of more than $150 \mathrm{~m}$ is injected into the ring, leaving a gap of less than $100 \mathrm{~m}$, which is maintained by four rf cavities during accumulation, to allow for extraction. In comparison, the transverse beam size is a few centimeters. In order to accumulate such an intense beam with low losses, the SNS has four horizontal and four vertical kicker magnets in the injection straight section to carry out detailed transverse beam painting. Proper programming of the waveforms of these kicker magnets is essential to paint a self-consistent, or any other, beam distribution into the SNS ring.

The remaining question for painting a self-consistent beam into the SNS is whether the injection kickers are capable of providing the necessary waveforms. The SNS injection scheme employs eight unipolar injection kickers: four in the horizontal plane and four in the vertical plane. We have already stated that the $x-y^{\prime}$ painting scheme allows a reasonable beam size to be painted without any kicker waveforms changing sign. However, it is still necessary to determine whether the kickers can provide sufficiently large kicks to create the required waveform throughout injection. The limiting factor turns out to be the second vertical injection kicker. In order to paint the necessary $y^{\prime}$ waveform at the stripper foil, it must provide a strong downward kick, as shown in the right-hand plot in Fig. 6. This kicker as installed is incapable of painting a sizable self-consistent beam using the $x-y^{\prime}$ painting scheme at the production energy of $1.0 \mathrm{GeV}$.

Fortunately, there are two further measures that can be taken to alleviate the constraint. The first is to lower the beam energy. The SNS has been operated with energies as low as $0.6 \mathrm{GeV}$, and at this energy a substantially larger kick can be achieved. The second is to create a closed orbit bump which is chosen to assist the second kicker to provide the necessary kick. The injection straight section in the SNS

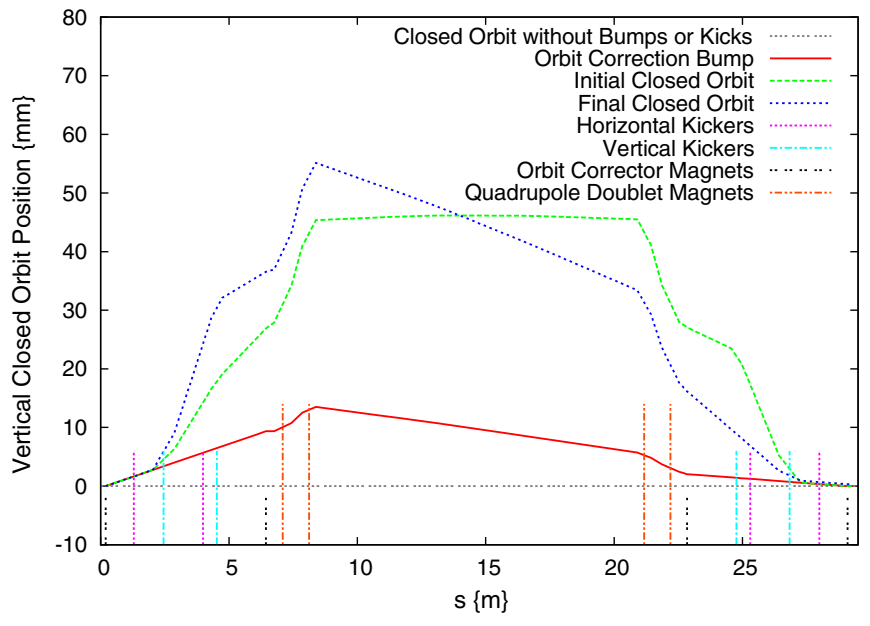

FIG. 10. Vertical closed orbit offsets in the injection straight section: (Red) orbit bump due to orbit correction dipoles, (green) initial closed orbit, and (blue) final closed orbit in the $x-y^{\prime}$ painting scheme. Also shown are the locations of the injection kickers, the orbit corrector magnets, and the quadrupole doublets. The stripper foil is located at the crossing point of the initial and final closed orbits.

ring has a pair of orbit corrector dipoles located near the ends of the straight section and another pair located just outside the quadrupole doublets (see Fig. 10). These can be used to create a closed bump in $y$ that moves the closed orbit closer to the stripper foil and also provides some of the negative slope in $y^{\prime}$ that is required in the $x-y^{\prime}$ painting scheme. Such a closed orbit bump modifies the injection kicker settings required to achieve a given value of $y^{\prime}$. This is illustrated in Fig. 10, which shows a vertical closed bump from the dipole corrector magnets in red together with the overall initial and final closed orbits in green and blue, respectively. This bump is totally achievable with the existing corrector magnets. Also shown in Fig. 10 are the locations of the injection kickers, the orbit corrector magnets, and the quadrupole doublet magnets. The stripper foil is located at the crossing point of the initial and final closed orbits.

If the injection energy is lowered to $0.6 \mathrm{GeV}$ and an appropriate closed orbit bump is used, it is possible to paint to a value of $y_{\text {Max }}^{\prime}=1.58 \mathrm{mr}$. Based on the resulting beam size and production parameters, this would correspond to an intensity of about $0.90 \times 10^{14}$ protons at a beam energy of $1.0 \mathrm{GeV}$. However, at $0.6 \mathrm{GeV}$, space charge forces are more intense, and so we propose to inject $0.35 \times 10^{14}$ protons at this energy. Consequently, the case that we now consider adopts this corrector-aided painting scheme with a maximum kick of $y^{\prime}=1.58 \mathrm{mr}$ at the stripper foil. We propose to inject an $0.6 \mathrm{GeV}$ beam for 300 turns to accumulate a maximum of $0.35 \times 10^{14}$ protons. We carry out this simulation using a pair of $0.5 \mathrm{~m}$ solenoid magnets set to $0.6 \mathrm{~T}$ magnetic field. The parameters for this case are summarized in Table I. 
TABLE I. Parameters for proposed self-consistent injection experiment.

\begin{tabular}{lc}
\hline \hline Tunes & $\left(Q_{x}, Q_{y}\right)=(6.18,6.18)$ \\
Beam energy & $E_{K}=0.6 \mathrm{GeV}$ \\
Injection & $0.35 \times 10^{14}$ protons in \\
& 300 turns \\
Solenoid field & $0.6 \mathrm{~T}$ \\
Dipole corrector kick & Maximum $=1.5 \mathrm{mrad}$ \\
Maximum painting & $x_{\mathrm{Max}}=34.4 \mathrm{~mm}$, \\
& $y_{\text {Max }}^{\prime}=1.58 \mathrm{mrad}$ in Eq. (1) \\
\hline \hline
\end{tabular}

We now describe the results of this simulation. We found that, following an initial offset due to the finite injected beam size, the rms transverse emittances increase linearly in time, as they should for self-consistent painting. Figure 11 shows the horizontal and vertical beam profiles at the injection point taken after 120 (left) and 300 turns (right). On top of the simulated beams, we have overlaid mathematical profiles for self-consistent beams. The results show that the cores of the simulated beams adhere very well to the self-consistent form, but the simulated profiles display wings that arise from nonlinearities, collective effects, finite injection spot size, and rf bunching. It is worth pointing out again that self-consistent beams have the desirable properties of uniform transverse density and small tune shift and tune spread. The agreement between the simulated and ideal self-consistent profiles at different times supports the argument that the painted beam is selfconsistent throughout injection.

Another check on self-consistency over time is shown in Fig. 12. The left-hand plot shows the averaged incoherent horizontal and vertical tunes as functions of the turn number. The near equality of these quantities over time is a good indicator of self-consistency. The right-hand plot shows the correlation coefficients of $x$ versus $y^{\prime}$ and $y$

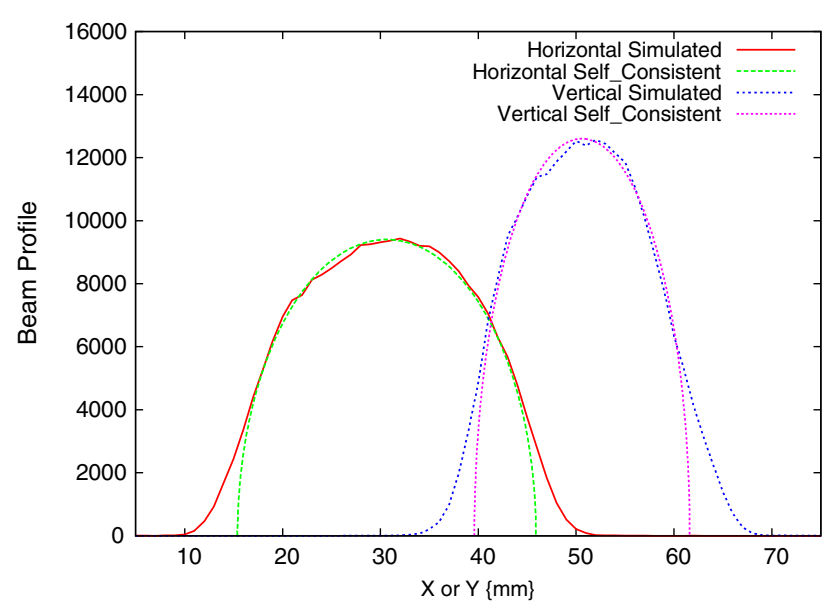

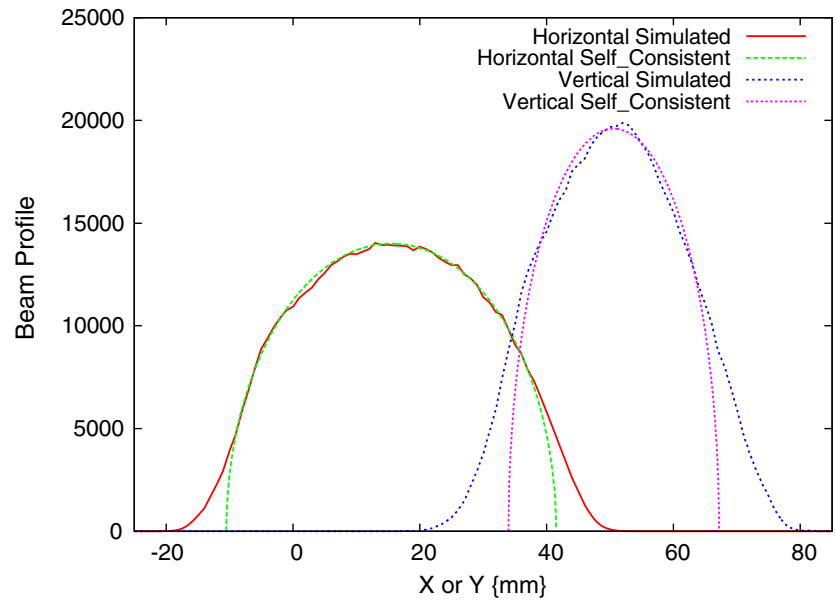

FIG. 11. Horizontal and vertical beam profiles taken after 120 (left) and 300 turns (right) of accumulation. Mathematically ideal profiles for self-consistent beams are overlaid on the simulation results.
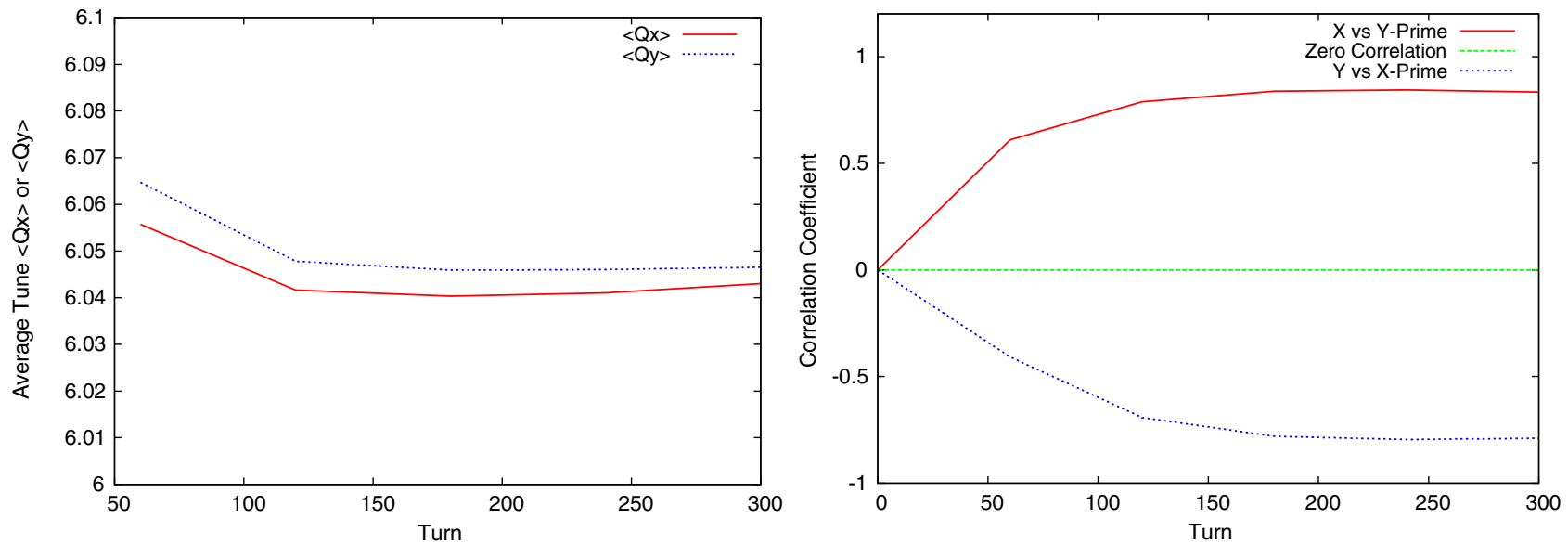

FIG. 12. (Left) Average horizontal and vertical incoherent tunes as functions of the turn number. (Right) Normalized correlation coefficients for $x$ versus $y^{\prime}$ and $y$ versus $x^{\prime}$ as functions of the turn number. The correlation coefficients are displayed for the beam at the injection location. 

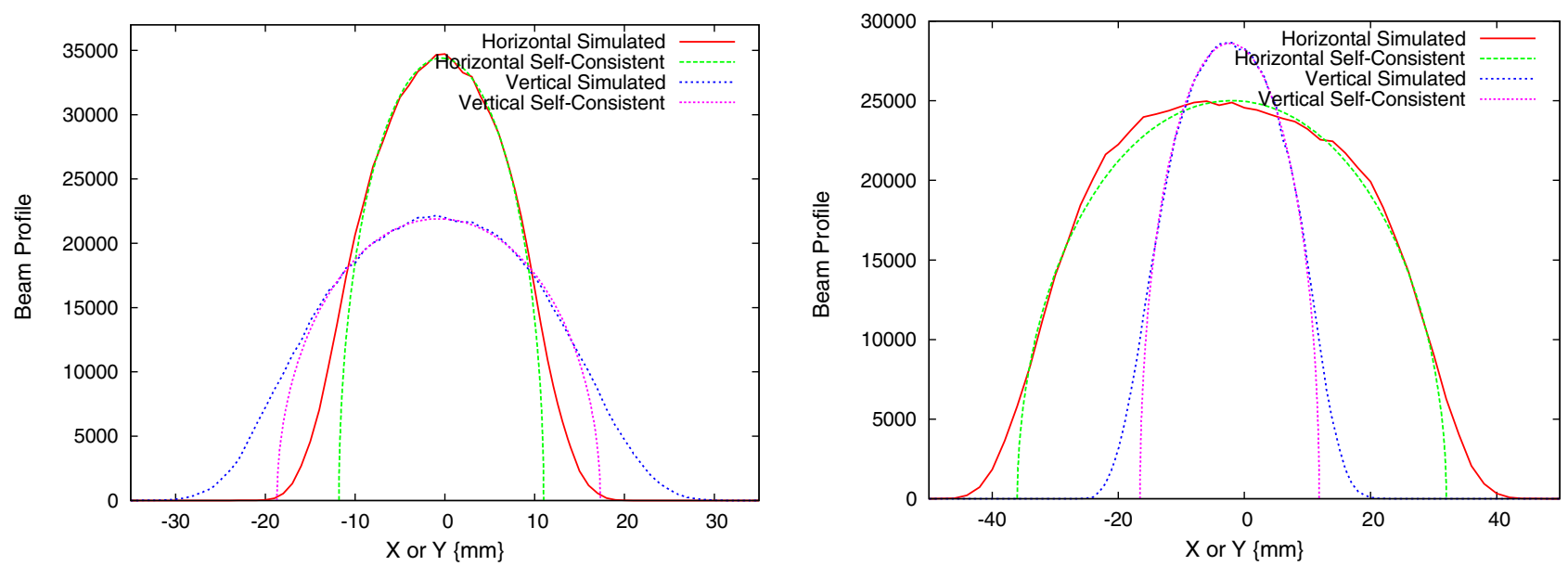

FIG. 13. Horizontal and vertical profiles of the accumulated beam taken at the locations of the electron scanner in the ring (left) and at wire scanner WS20 in the RTBT (right). A comparison with the superimposed self-consistent profiles indicates that the accumulated beam demonstrates self-consistent profile behavior.

versus $x^{\prime}$ taken at the injection location as functions of the turn number. Figure 12 shows that the correlations between $x$ and $y^{\prime}$ and between $y$ and $x^{\prime}$ become very strong after about 50 turns. By comparison, the correlation coefficients between all other transverse variables in this calculation have absolute values less than 0.15 , and a similar calculation carried out using the standard SNS correlated (non-self-consistent) painting yields transverse correlation coefficient absolute values less than 0.07 . The reason that results are displayed at 120 turns and at 300 turns in Fig. 11 is that, during the process of painting a self-consistent beam, the beam must be self-consistent throughout the process and not just at the end. This can be seen by comparing the profiles in Fig. 11 at 120 turns with those at 300 turns. It can also be seen in the results that are plotted in Fig. 12. Finally, we note that this case is within the capabilities of the present SNS ring, assuming the solenoid magnets have been installed.

\section{OBSERVATION AND CONFIRMATION OF A SELF-CONSISTENT BEAM}

The above results demonstrate that it should be possible to paint a self-consistent rotating distribution into a highintensity ring. However, there is little point in doing so unless the self-consistency can be confirmed. The results presented above were all derived from evaluations performed at the location of injection, where the correlations between $x-y^{\prime}$ and $y-x^{\prime}$ hold. Unfortunately, in the case of the SNS, observations cannot be made at this location. The diagnostics in the SNS that can be used to observe injected beams include five wire scanners and a multiwire profile monitor (harp) in the ring to target beam transport line (RTBT), which carries the extracted beam from the ring to the target, and an electron scanner in the ring [23-25], which can be used to make non-destructive profile measurements during accumulation. Although the details of the correlations that are associated with self-consistency change as the beam is transported through channels where the vertical and horizontal phase advances differ, the beam will remain self-consistent in the limit of all linear transport. This implies that the beam should remain elliptical with a constant density, meaning that the beam profiles should display self-consistent behavior. Also, because the correlations are transported with the beam, it should be possible to observe strong correlations in various transverse quantities, with the details depending on the location. Figure 13 shows horizontal and vertical profiles for an accumulated beam transported to the location of the electron scanner (left) in the ring and alternatively transported to the wire scanner WS20 (right) in the RTBT. In both cases, a comparison shows that the simulated results agree with superimposed self-consistent profiles. It is a straightforward task to measure these profiles experimentally.

The point raised above regarding correlations is illustrated in Table II. The table shows the correlation coefficients for $x-y, x-y^{\prime}, y-x^{\prime}$, and $x^{\prime}-y^{\prime}$ at the locations of the foil (ring), the electron scanner (ring), wire scanners WS02, WS20, WS21, WS23, and WS24, and the harp (all in the RTBT). The coefficients $c_{x x^{\prime}}$ and $c_{y y^{\prime}}$ are not shown, because these are simply related to the Courant-Snyder $\alpha$ parameters and are, therefore, not very useful. Table II shows that these quantities vary from strongly to weakly correlated depending on the relationship of the horizontal and vertical phases at the location where they are observed. The measurement of these correlations in addition to the beam profiles will provide strong evidence that the beam is self-consistent.

With horizontal, vertical, and diagonal wire scanners, such as those available in the RTBT, several quadrupole scans can be used to reconstruct the full 4D sigma matrix even in the absence of coupling elements [26]. From the 
TABLE II. Correlation coefficients from simulations at locations of diagnostics.

\begin{tabular}{lrrrr}
\hline \hline Location & \multicolumn{1}{c}{$c_{x y}$} & \multicolumn{1}{c}{$c_{x y^{\prime}}$} & \multicolumn{1}{c}{$c_{y x^{\prime}}$} & \multicolumn{1}{c}{$c_{y^{\prime} x^{\prime}}$} \\
\hline Foil & -0.12 & 0.75 & -0.67 & 0.03 \\
Electron scanner & 0.42 & 0.77 & -0.62 & -0.73 \\
Wire scanner 02 & 0.43 & 0.71 & -0.61 & -0.69 \\
Wire scanner 20 & 0.73 & 0.33 & -0.73 & -0.18 \\
Wire scanner 21 & 0.59 & -0.32 & 0.18 & 0.42 \\
Wire scanner 23 & 0.70 & -0.14 & 0.59 & 0.04 \\
Wire scanner 24 & 0.78 & 0.71 & -0.72 & -0.50 \\
Harp & -0.45 & 0.63 & -0.54 & 0.65 \\
\hline \hline
\end{tabular}

sigma matrix, one can extract the correlation coefficients to compare with those shown in Table II. This technique was developed further [27], optimizing the measurement by using several quadrupoles for more complete control over the phase advance. We are currently investigating the RTBT optics that will be used for this measurement.

We have demonstrated that, in the SNS, it is possible to conduct measurements that will strongly determine whether a beam is self-consistent. The analysis suggests that the injection of a self-consistent beam into the SNS ring can be accomplished with only the addition of solenoids having an integrated strength of $\sim 0.6 \mathrm{~T}-\mathrm{m}$. These solenoid magnets can be installed in the rf straight section of the ring symmetrically at locations having roughly equal horizontal and vertical beta functions, although there is considerable flexibility on the exact configuration and positioning of the magnets. We plan to study experimentally the case described here to demonstrate the painting of a selfconsistent beam in the SNS.

\section{SUMMARY}

This paper has presented the results of extensive computational studies performed to demonstrate the feasibility of painting a rotating uniform-density self-consistent beam into a high-intensity ring, using the SNS accumulator as an example. The calculations were performed using realistic physics models and a detailed representation of the ring configuration. In particular, we employed symplectic transport with fringe fields, space charge, transverse and longitudinal impedances, injection and foil scattering, $\mathrm{rf}$ focusing, and beam loss due to a complete set of apertures and collimators. We demonstrated that, if solenoids are present to break the rotating and counterrotating beam degeneracy, then nonlinearities and collective effects do not destroy self-consistency. If the rf can supply a uniform longitudinal beam profile, then the coasting beam assumption necessary for painting a rotating distribution is valid. We developed an extended fringe field tracking model that was used for the quadrupole doublets in the injection straight section, which are traversed well off axis by the circulating beam during painting. We examined a variety of possible painting scenarios to determine whether they would lead to acceptable losses. For the SNS, this led to the adoption of an $x-y^{\prime}$ painting scheme that was applied to all following work.

To determine the robustness of the self-consistent painting, we studied sensitivity to the solenoid field strength and placement and to the lattice tune separation. Results of these calculations show that there is not a great sensitivity to any of these factors that would impact the possibility of painting a rotating beam.

We next considered the prospect of painting a rotating beam into the SNS accumulator ring. Although the achievable strength of the second vertical kicker is a limiting factor, we found that, by lowering the beam energy and utilizing orbit corrector dipoles in the injection straight section, it is possible to paint a self-consistent rotating distribution into the SNS ring.

We also discussed what measurements can be made to validate the self-consistency of the painted beam. In the SNS, the existing diagnostic hardware, consisting of wire scanners and a multiwire profile monitor in the RTBT transport line and an electron scanner located in the ring, can be used to provide profile measurements and correlation functions between transverse variables that will strongly support the demonstration of self-consistency.

The only hardware change that we envision in order to carry out the proposed experiment is the installation of solenoid magnets of integrated field strength of $\sim 0.6 \mathrm{~T}-\mathrm{m}$ in the straight sections of the ring. As a result, we conclude that it is feasible to paint a rotating self-consistent distribution into the SNS ring. Even though nonlinear effects and a finite painted beam size distort the profiles from ideal mathematical self-consistency at the edges, the computational results yield beams of more uniform and lower transverse density than are found for the present correlated SNS painting scheme. Such beams are very desirable for high-intensity, fixed target accelerators such as the SNS.

Finally, even though the work presented here was closely tied to the specifics of the SNS ring, the issues considered will be confronted in any effort to paint a self-consistent beam into a ring, and we believe it is useful to present these considerations as illustrations of the process.

\section{ACKNOWLEDGMENTS}

This research was supported by the DOE Office of Science, Basic Energy Science, Accelerator and Detector Research Program.

[1] M. Reiser, Theory and Design of Charged Particle Beams, Wiley Series in Beam Physics and Accelerator Technology (John Wiley and Sons, Inc., New York, 1994).

[2] I. M. Kapchinsky and V. V. Vladimirsky, Limitations of proton beam current in a strong focusing linear accelerator 
with the beam space charge, in Proceedings of the Conference on High Energy Accelerators and Instrumentation (CERN, Geneva, 1959), p. 274.

[3] F. J. Sacherer, Transverse space charge effects in circular accelerators, Ph.D. thesis, University of California, Berkeley, CA, 1968.

[4] I. Hofmann, L. J. Laslett, L. Smith, and I. Haber, Stability of the Kapchinskij-Vladimirskij (K-V) distribution in long periodic transport systems, Part. Accel. 13, 145 (1983).

[5] Yu. K. Batygin, Adiabatic matching of a nonuniform intense charged-particle beam into the focusing channel, Phys. Rev. E 54, 5673 (1996).

[6] R. L. Gluckstern, A. V. Fedotov, S. S. Kurennoy, and R. D. Ryne, Longitudinal halo in beam bunches with selfconsistent 6-D distributions, in Proceedings of Workshop on Space Charge Physics in High Intensity Hadron Rings, Shelter Island, NY, 1998, AIP Conference Proceedings Vol. 448 (AIP, Woodbury, NY, 1998).

[7] R. C. Davidson, H. Qin, S. I. Tsenov, and E. A. Startsev, Kinetic description of intense beam propagation through a periodic focusing field for uniform phase-space density, Phys. Rev. ST Accel. Beams 5, 084402 (2002).

[8] V. V. Danilov and E. A. Perevedentsev, Two examples of integrable systems with round colliding beams, in Proceedings of the Particle Accelerator Conference, Vancouver, BC, Canada, 1997 (IEEE, New York, 1998), p. 1759.

[9] V. Danilov, S. Cousineau, S. Henderson, and J. Holmes, Self-consistent time dependent two dimensional and three dimensional space charge distributions with linear force, Phys. Rev. ST Accel. Beams 6, 094202 (2003).

[10] O. J. Luiten, S. B. van der Geer, M. J. de Loos, F. B. Kiewiet, and M. J. van der Wiel, How to Realize Uniform Three-Dimensional Ellipsoidal Electron Bunches, Phys. Rev. Lett. 93, 094802 (2004).

[11] P. Musumeci, J. T. Moody, R. J. England, J. B. Rosenzweig, and T. Tran, Experimental Generation and Characterization of Uniformly Filled Ellipsoidal Electron-Beam Distributions, Phys. Rev. Lett. 100, 244801 (2008).

[12] J. A. Holmes, S. Cousineau, V. V. Danilov, S. Henderson, A. Shishlo, Y. Sato, W. Chou, L. Michelotti, and F. Ostiguy, ORBIT: Beam dynamics calculations for high-intensity rings, in The ICFA Beam Dynamics Newsletter, Vol. 30 (ICFA Beam Dynamics Panel, 2003), p. 100.

[13] V. Danilov, S. Cousineau, S. Henderson, J. Holmes, and M. Plum, Injection schemes for self-consistent space charge distributions, in Proceedings of the European Particle Accelerator Conference (EPAC04), Lucerne, Switzerland, 2004 (EPS-AG, Lucerne, 2004).

[14] J. Holmes, V. Danilov, and S. Cousineau, Painting selfconsistent beam distributions in rings, in Proceedings of the 33rd ICFA Advanced Beam Dynamics Workshop on High Intensity and High Brightness Hadron Beams Bensheim, Germany, 2004 (ICFA Beam Dynamics Panel, Bensheim, Germany, 2004).
[15] J. Holmes, S. Cousineau, and V. Danilov, Painting selfconsistent beam distributions in rings, in Proceedings of the 21st Particle Accelerator Conference, Knoxville, TN, 2005 (IEEE, Piscataway, NJ, 2005).

[16] J. A. Holmes, S. M. Cousineau, V. V. Danilov, and A. P. Shishlo, RF barrier cavity option for the SNS ring beam power upgrade, in Proceedings of the 39th ICFA Advanced Beam Dynamics Workshop on High Intensity and High Brightness Hadron Beams, Tsukuba, Japan, 2006 (ICFA Beam Dynamics Panel, Tsukuba, Japan, 2006).

[17] E. Forest, Beam Dynamics, A New Attitude and Framework (Harwood Academic, Amsterdam, 1998).

[18] OPERA3D/TOSCA, developed by Vector Fields Limited, England.

[19] J. G. Wang, Particle optics of quadrupole doublet magnets in Spallation Neutron Source accumulator ring, Phys. Rev. ST Accel. Beams 9, 122401 (2006).

[20] J. D. Galambos, S. Danilov, D. Jeon, J. A. Holmes, D. K. Olsen, F. Neri, and M. Plum, Comparison of simulated and observed beam profile broadening in the Proton Storage Ring and the role of space charge, Phys. Rev. ST Accel. Beams 3, 034201 (2000).

[21] S. Cousineau, S. Y. Lee, J. Holmes, V. Danilov, and A. Fedotov, Space charge induced resonance excitation in high intensity rings, Phys. Rev. ST Accel. Beams 6, 034205 (2003).

[22] S. Cousineau, J. Holmes, J. Galambos, A. Fedotov, J. Wei, and R. Macek, Resonant beam behavior studies in the Proton Storage Ring, Phys. Rev. ST Accel. Beams 6, 074202 (2003).

[23] W. Blokland, Non-invasive beam profile measurements using an electron-beam scanner, in Proceedings of the 46th ICFA Advanced Beam Dynamics Workshop on HighIntensity and High-Brightness Hadron Beams HB2010, Morschach, Switzerland, 2010 (PSI, Viligen, Switzerland, 2011).

[24] A. Aleksandrov, S. Assadi, S. Cousineau, V. Danilov, M. Plum, P. Logatchov, and A. Starostenko, Feasibility study of using an electron beam for profile measurements in the SNS accumulator ring, in Proceedings of the 21st Particle Accelerator Conference, Knoxville, TN, 2005 (IEEE, Piscataway, NJ, 2005).

[25] W. Blokland and S. Cousineau, A non-destructive profile monitor for high intensity beams, in Proceedings of the 24th Particle Accelerator Conference, PAC-2011, New York, 2011 (IEEE, New York, 2011).

[26] P. Raimondi, P. Emma, N. Toge, N. J. Walker, and V. Ziemann, Sigma matrix reconstruction in the SLC final focus, in Proceedings of the 15th Particle Accelerator Conference, PAC-1993, Washington, DC, 1993 (IEEE, New York, 1993).

[27] E. Prat and M. Aiba, Four-dimensional transverse beam matrix measurement using the multiple-quadrupole scan technique, Phys. Rev. ST Accel. Beams 17, 052801 (2014). 